Instituto Internacional de Investigación y Desarrollo Tecnológico Educativo INDTEC, C.A.

DOI: https://doi.org/10.29394/Scientific.issn.2542-2987.2021.6.21.3.58-80

OAI-PMH: http://www.indteca.com/ojs/index.php/Revista Scientific/oai

Artículo Original / Original Article

\title{
Gestión de información: eslabón imprescindible para la efectiva comunicación en el comercio exterior
}

\author{
Autores: César Raúl Méndez Carpio \\ Universidad Católica de Cuenca, UCACUE \\ cmendezc@ucacue.edu.ec \\ Cuenca, Ecuador \\ https://orcid.org/0000-0003-0582-0107 \\ Yunier Rodríguez Cruz \\ Universidad de La Habana, UH \\ yunier@fcom.uh.cu \\ La Habana, Cuba \\ https://orcid.org/0000-0002-8982-1256 \\ Hilda María Saladrigas Medina \\ Universidad de La Habana, UH \\ saladrigas@fcom.uh.cu \\ La Habana, Cuba \\ https://orcid.org/0000-0001-5601-8267
}

\section{Resumen}

Las empresas de diferente nicho de mercado, desarrollan la prestación de servicios y/o productos con miras a la internacionalización a través del comercio exterior, varias modelan sistemas de gestión por procesos considerando prácticas de eficacia y eficiencia propendiendo la seguridad de la carga como son las normas Basic Alliance for Secure Commerce (BASC). Este estudio, pretende analizar la integración de características y preceptos teóricos del manejo de información para una efectiva comunicación en las empresas de comercio exterior que aplican estas normas y proponer su innovación normativa. Se desarrolló una metodología de enfoque cualitativo de tipo descriptivo apoyado en la revisión bibliográfica y se obtuvo como principal resultado que, en las organizaciones, se tratan de manera aislada a la gestión de información y la comunicación, por lo que se consiguen estudios parciales y al no estar integrados todos los elementos informacionales, la comunicación no es efectiva; lo que presume, la materialización de riesgos y el cometimiento de actividades ilícitas por parte de personas o grupos inescrupulosos externos al negocio, problema que puede ser mitigado con la actualización normativa en los datos de entrada y elementos de la salida de la revisión por parte de la dirección empresarial.

Palabras clave: información; gestión de información; comunicación; gestión de comunicación; comercio exterior.

Código de clasificación internacional: 5310.09 - Relaciones comerciales internacionales.

Cómo citar este artículo:

Méndez, C., Rodríguez, Y., \& Saladrigas, H. (2021). Gestión de información: eslabón imprescindible para la efectiva comunicación en el comercio exterior. Revista Scientific, 6(21), 58-80, e-ISSN: 2542-2987. Recuperado de: https://doi.org/10.29394/Scientific.issn.25422987.2021.6.21.3.58-80

Fecha de Recepción: 24-03-2021
Fecha de Aceptación: 01-07-2021
Fecha de Publicación: 05-08-2021 
OAI-PMH: http://www.indteca.com/ojs/index.php/Revista Scientific/oai

Artículo Original / Original Article

\title{
Information management: essential link for effective communication in foreign trade
}

\begin{abstract}
The companies of different market niche, develop the provision of services and / or products with a view to internationalization through foreign trade, several model management systems by processes considering practices of effectiveness and efficiency, promoting cargo security such as Basic Alliance for Secure Commerce (BASC) standards. This study aims to analyze the integration of characteristics and theoretical precepts of information management for effective communication in foreign trade companies that apply these standards and propose their regulatory innovation. A qualitative, descriptive approach methodology was developed supported by the bibliographic review and the main result was that, in organizations, information management and communication are treated in isolation, for which partial studies are obtained and the not being integrated all the informational elements, the communication is not effective; what it presumes, the materialization of risks and the commission of illegal activities by unscrupulous people or groups outside the business, a problem that can be mitigated with the regulatory update in the input data and elements of the output of the review by business management.
\end{abstract}

Keywords: information; information management; communication; communication management; foreign trade.

International classification code: 5310.09 - International trade relations.

How to cite this article:

Méndez, C., Rodríguez, Y., \& Saladrigas, H. (2021). Information management: essential link for effective communication in foreign trade. Revista Scientific, 6(21), 58-80, e-ISSN: 2542-2987. Recovered from: https://doi.org/10.29394/Scientific.issn.2542-2987.2021.6.21.3.58-80

Date Received:

24-03-2021
Date Acceptance:

01-07-2021
Date Publication: 05-08-2021 


\section{Introducción}

El comercio exterior es la fuente de desarrollo de muchos países en el mundo y, su difusión ha permitido posicionar a las empresas a través de la venta de sus productos, en una escala de importancia dentro del contexto internacional. Esta práctica cada día se vuelve más exigente y competitiva por lo que grupos antisociales ven en esta actividad un medio o canal de corrupción para concretar sus propósitos antiéticos en desmedro del comercio internacional.

Las innovaciones y las buenas prácticas, han permitido destacar en el tiempo y en las diversas relaciones comerciales, el desarrollo de normas y tendencias que controlan las actividades ilícitas producto de las malas prácticas que se destacan hoy en día en el comercio exterior como son: el tráfico de sustancias estupefacientes, lavado de activos, corrupción, soborno, terrorismo, entre otros.

Las normas Basic Alliance for Secure Commerce (BASC), con la aplicación de sus acápites, intentan controlar o disminuir la materialización de las actividades ilícitas por lo con esta investigación, se ha considerado analizarlas con el fin de verificar si entre sus preceptos teóricos han sido consideradas todas las características de la gestión de la información que permitan una comunicación efectiva, debiendo además plantear una mejora para la toma de decisiones de la alta dirección

\subsection{La Gestión de Información y la Comunicación en el comercio exterior ecuatoriano}

Al ser la información una parte constitutiva de toda organización, debe ser analizada y gestionada de manera adecuada y completa para conseguir el propósito en función de los objetivos estratégicos dependiendo su proyección en el mercado en el que se desenvuelvan. Este enfoque lo mencionan Quintero, Giraldo, Bernal, Viana y Taborda (2009): y lo tratan "Como ciencia 
interdisciplinaria que comprende los esfuerzos y habilidades de distintos profesionales, con una función teórica y científica alrededor de la información, tiene conexiones con los estudios sobre los procesos de comunicación [...]" (pág. 206); por tanto, es de suma importancia analizar y comprender sus elementos o componentes.

En concordancia con ello y resumiendo, es relevante aplicar el criterio que plantea Rodríguez (2015): cuando trata a la gestión de la información como proceso estratégico de decisión y menciona que en las organizaciones se pueden identificar sistemas de información a nivel estratégico, administrativo y operativo coincidiendo que el carácter informacional de la toma de decisiones viene dado por:

- El ambiente informacional que se genera en la toma de decisiones respecto a la importancia y el rol de la información).

- Las Fuentes documentales, personales e institucionales de Información que promueven la ejecución de los procesos.

- La toma de decisiones basada en la ejecución de los procesos de búsqueda, selección, procesamiento, análisis e interpretación.

- El análisis de su sistema de Información que permite viabilizar los procesos informacionales.

- La forma en que se comportan los individuos frente al uso de información, los procesos y sistemas.

Si se analizan estos componentes y se los define en los objetivos estratégicos de la empresa, tendremos como resultado, un conjunto de estrategias ancladas a la gestión organizacional y su desarrollo.

Con lo dicho se debe reconocer la participación activa de la gerencia en el despliegue de los objetivos estratégicos de la empresa que como indica Medina (2019): una buena gestión debe basarse e: 
[...] El liderazgo del talento humano para posesionarse de las decisiones que correspondan a los requerimientos sociales para determinar la realidad organizacional, de tal manera de poder prever el futuro, que permitan lograr de las metas deseadas en los ámbitos inmediatos de desarrollo sustentable (pág. 277).

En consecuencia, con la gestión de información y la participación activa de la gestión estratégica de las empresas, Gomes y Pomim (2010): concluyen en su estudio que "la información en el contexto actual asume un papel importante, proporcionando al entorno empresarial una actuación dinámica, en constante renovación y adaptarse al entorno en el que opera" (pág. 117).

En este entorno se mueven las empresas de comercio exterior y es importante anotar que, la información debe estar controlada en toda la cadena logística, priorizar la seguridad en los flujos de información y en la entrega de los servicios de información permitiendo que funcione como un sistema anclado a la planificación estratégica y que incluya, delegación de responsabilidades y alfabetización informacional controlados por actividades de auditoría interna para enfocar los impactos en beneficio del desarrollo organizacional.

Por otro lado, la comunicación en toda organización y en especial en las empresas de comercio exterior, tiene un carácter primordial tanto al interior de la empresa para transmitir los mensajes a través de canales de comunicación de las actividades propias del giro del negocio; como al exterior de la misma, para conseguir llegar a los asociados de negocios con el fin de vender un producto y posicionarse en el mercado internacional obteniendo una imagen determinada y corporativa.

Al ser considerada, un proceso intangible dentro de las organizaciones, los componentes de la comunicación como son: los públicos, los canales, los flujos, las redes e incluso los recursos de la comunicación, en muchas organizaciones de comercio exterior, se gestionan y controlan de forma aislada 
por lo que sus efectos son parciales. En este sentido, Fernández y Useche (2015), consideran que:

La comunicación en la organización es una herramienta fundamental para que los públicos internos conozcan sus funciones y más allá de ejercerlas por obligación se sientan comprometidos y motivados con y por la empresa; entiendan la misión, visión, filosofía y, además, las compartan como propia, generándose así una cultura y un clima organizacional armónico (págs. 63-64).

Como se afirma en este concepto, únicamente se analizan los públicos internos y su relación con la empresa más se debería considerar también los públicos externos o asociados de negocios y que se controle el impacto de la comunicación en el comercio exterior puesto que este análisis determinaría el futuro de las organizaciones que se dedican a este negocio.

Las empresas de comercio exterior en efecto deben dirigir sus esfuerzos a complementar los procesos comunicacionales, gestionando con calidad sus públicos externos lo que permitirá tener el control y el impacto en la trazabilidad de sus cargas frente a sus asociados de negocios.

\subsection{Normativas de seguridad de la carga en el comercio internacional BASC}

Las normas Basic Alliance for Secure Commerce (BASC) nacen con la necesidad de tener apoyo para enfrentar procesos de contaminación de drogas y otras actividades ilícitas que se presentan como consecuencia de las actividades producto del comercio exterior. Estas normas, gestionan su actividad enfocada a la seguridad en los procesos y se fundamentan en nueve capítulos que son: 1). Objeto y alcance; 2). Referencias normativas; 3). Términos y definiciones; 4). Contexto de la empresa; 5). Liderazgo; 6). Planificación; 7). Apoyo; 8). Evaluación del desempeño; y 9). Mejora. 
OAI-PMH: http://www.indteca.com/ojs/index.php/Revista Scientific/oai

Artículo Original / Original Article

\subsection{Actividades ilícitas en el comercio exterior y análisis de vulnerabilidades}

La región latinoamericana es muy vulnerable a los efectos de las actividades ilícitas ya que al considerarse lucrativas se complementan con actividades de carácter criminal como: el comercio de ilegal de personas, armas y la materialización del narcotráfico. Este fenómeno se considera hoy en día como de carácter regional y fronterizo ya que el Ecuador es considerado un país de paso al tráfico de drogas mundial.

El poder económico acumulativo que deja a las personas dedicadas a este tipo de comercio ilegal, se alimenta de un fenómeno sociológico de aceptación de las personas que viven especialmente en fronteras y espacios de siembra y cosecha que lo ven como "normal" y como parte de su forma de vida diaria.

Los países de la zona, han tratado de frenar y mitigar los riesgos producto de estos procesos y es así que adopta normas y procedimientos que apoyen a la seguridad de la información coadyuvante al comercio de productos a nivel internacional. Así mismo se generan otras actividades ilícitas producto de los procesos productivos internacionales como es el lavado de activos.

Las normas Basic Alliance for Secure Commerce (BASC, 2017a), puntualizan los tipos de actividades ilícitas y enfocan su finalidad al establecerse como:

[...] Cultura de seguridad a través de la cadena de suministro, mediante la implementación de sistemas de gestión e instrumentos aplicables al comercio, sectores relacionados y otras empresas que deseen gestionar los controles operacionales básicos que le permitan una operación segura (pág. 3).

Dentro de la estructura articular de las normas de seguridad mencionadas, se presentan ciertas características que incluyen algunos elementos tanto de información como de la comunicación y que tienen como 
propósito dar seguridad al tratamiento de la carga internacional; dichos elementos no son tratados completamente por lo que se considera de suma importancia la inclusión de todos los componentes de la gestión de información desde que se genera el producto o servicio, hasta su destino, incentivando una efectiva comunicación y favoreciendo la trazabilidad en el manejo de la carga del comercio exterior; por lo tanto, la gestión de información como la gestión de la comunicación adecuadas, facilitarán la ejecución de los procesos sustantivos en las empresas que se encuentran inmersas en la cadena logística o llamada cadena de suministros del comercio exterior.

Los directivos de las empresas en mención, deben estar atentos a los cambios y a la mejora; así como tener en cuenta una adecuada percepción de los criterios o elementos de la información y estar consecuentes con los conocimientos que mejoren la comunicación y su gestión Es consecuencia con la percepción organizacional y la importancia de la información en las empresas de este sector, Sánchez-Castellano, Rodríguez-Cruz y Río-López (2018), manifiestan que:

La percepción organizacional necesita un adecuado uso de información en los diferentes niveles de decisión; condiciona todo el accionar estratégico y operativo de la organización, así como la forma en que sus miembros comparten y socializan visiones, significados e interpretaciones que hacen del ambiente externo e interno (pág. 6).

A más del uso adecuado de la información, la comunicación imbrica un papel complementario e importante que debe estar dentro de toda organización como parte de su conocimiento para la mejora; es así como Rueda, Tamayo, Acosta, Cueva y Idrobo (2020): mencionan que "cualquier organización que quiera transformarse en una organización que aprende necesita la intervención de la comunicación para actuar como medio de formación, transmisión, difusión y distribución de los nuevos valores culturales que determinen el comportamiento nuevo de las personas" (pág. 83). 
El objetivo de este estudio, pretende incluir en la estructura de las normas BASC, los elementos de la Gestión de información como un eslabón imprescindible para conseguir una efectiva comunicación en las empresas de comercio exterior.

\subsection{El Comercio Exterior}

Para varios países del mundo, el comercio internacional es considerado prioritario, por haberse convertido en la base de desarrollo económico. Esta actividad empresarial conocida como comercio exterior por lo tanto busca la internacionalización de la industria a través de la apertura comercial entre los países, el incremento de la productividad, la mejora en la calidad de los productos siendo cada vez más competitivos en los mercados internacionales. En consecuencia, con este criterio, Huesca (2012), señala que el comercio exterior es:

En esencia el intercambio de bienes, servicios y conocimientos entre las naciones que integran el planeta, son una fuerza que permite el desarrollo económico, y promueve el bienestar de las naciones y de su población, que bienes que se comercializan están constituidos por bienes terminados, intermedios, que sirven para elaborar otros productos y materias primas (pág. 15).

\subsection{Características del comercio exterior o internacional}

El comercio exterior tiene como finalidad el intercambio de productos y/o servicios entre distintos países, su origen se genera ya que, al interior de los países, la cantidad de productos elaborados, no son consumidos en su totalidad por lo que se requiere ofrecer a comunidades externas que no los producen.

La comunidad mundial económica reconoce a esta actividad como comercio internacional basada en la organización responsable de intercambio de bienes y servicios dedicados a la exportación e importación de estos. Es 
así que día a día aparecen nuevos mercados y con ellos un conjunto de leyes y normativas que intentan regular su actividad comercial convirtiendo a este desempeño económico como una parte muy esencial y estructural de sus desarrollos productivos.

Para Ponce (2005): contribuye al análisis recordando que; las políticas arancelarias son consideradas como el manejo de instrumentos que el estado tiene a su alcance para regular las relaciones comerciales con el resto del mundo y mantener el desarrollo de un sistema capitalista aplicado. Además, mediante estas políticas, los estados impulsan la protección a sus mercados internos nacionales cumpliendo positivamente con su función de recaudar ingresos fiscales lo que se reconoce como estados proteccionistas.

Es preciso reconocer que el comercio internacional es influenciado de manera directa por las políticas comerciales de los países y de ello depende la fluidez de este tipo de comercio, así lo menciona Salvatore (2005): quien afirma también que, a través del implemento de aranceles y subvenciones que causan impacto en los procedimientos y que en algunos casos son consideradas como barreras arancelarias que en varios casos tratan de proteger a los productores nacionales que pueden quedar en desventaja frente a las exportaciones. Así mismo estas políticas arancelarias intentan minorar la competencia desleal con el fin de equilibrar la balanza comercial al disminuir la cantidad de importaciones con el objetivo de incrementar los ingresos fiscales como parte esencial del presupuesto nacional.

\section{Metodología (Materiales y métodos)}

La metodología relacionada con esta investigación, se centró en el enfoque cualitativo a nivel descriptivo y tuvo refuerzo en la técnica de revisión bibliográfica, analizando los requisitos normativos Basic Alliance for Secure Commerce (BASC) en concordancia con los temas de información y su incidencia en la comunicación verificando para luego identificar los elementos 
de la gestión de información, así como los componentes de la gestión de la comunicación enfocados a las empresas de comercio exterior certificadas con dichas normas, verificando la completa integración de los mismos.

Los referentes conceptuales consideraron varios autores y en diferentes revistas indexadas que han servido de marco teórico y de guía para la investigación propuesta. La muestra de estudio tuvo relación directa con empresas que forman parte del capítulo BASC en el Ecuador y que están en la línea del comercio internacional; siendo estas: exportadores e importadores, transportistas de carga, agentes de aduana y de carga y navieras.

Para el proceso de recolección de datos, se diseñó una encuesta estructurada la misma que se aplicó a gerentes de procesos y representantes de las empresas seleccionadas a través de la plataforma de Google Drive y que consideró los elementos de la gestión de información y los componentes de la gestión de la comunicación en relación con la aplicación de estos temas en sus procedimientos mandatorios.

\section{Resultados de la investigación}

En la actualidad las normas Basic Alliance for Secure Commerce (BASC, 2017b): acerca de la revisión por parte de la alta dirección para la toma de decisiones, considera a la información únicamente como datos de entrada y a la comunicación como elementos de salida.

Con lo mencionado vs la teoría de gestión de información se evidencia carencia de varios elementos tanto de la gestión de información como de la gestión de la comunicación; por lo que, fue necesario aplicar una encuesta de seguimiento con el fin de conocer sobre elementos informacionales que pudieran estar tratados de forma intrínseca en la gestión estratégica, operativa o de apoyo en las actividades propias de las organizaciones que formaron parte de la investigación y que no se reportan en los ítems de las normas; así mismo componentes de la gestión de la comunicación relevantes en el 
contexto del comercio exterior. La tabla 1, expone los resultados obtenidos producto de la aplicación de la encuesta mencionada.

Tabla 1. Gestión de información e incidencia en la efectiva comunicación en empresas que utilizan BASC en el comercio exterior.

\begin{tabular}{|c|c|c|}
\hline $\begin{array}{l}\text { COMPONENTES DE LA GESTIÓN DE INFORMACIÓN Y GESTIÓN DE } \\
\text { LA COMUNICACIÓN }\end{array}$ & SI & NO \\
\hline $\begin{array}{l}\text { 1. ¿La organización dispone de un sistema de información para controlar } \\
\text { fuentes de información importante? }\end{array}$ & $20 \%$ & $80 \%$ \\
\hline 2. ¿La gestión por procesos incluye el uso adecuado de la información? & $20 \%$ & $80 \%$ \\
\hline $\begin{array}{l}\text { 3. ¿Cree Usted que se controla la información que se entrega a sus } \\
\text { asociados de negocios? }\end{array}$ & $80 \%$ & $20 \%$ \\
\hline $\begin{array}{l}\text { 4. ¿Las competencias del personal son adecuadas para el manejo de la } \\
\text { información? }\end{array}$ & $50 \%$ & $50 \%$ \\
\hline $\begin{array}{l}\text { 5. ¿El plan de capacitación incluye competencias informacionales en el } \\
\text { desarrollo del personal? }\end{array}$ & $20 \%$ & $80 \%$ \\
\hline 6. ¿Considera que se revisan los resultados de la Gestión informacional? & $20 \%$ & $80 \%$ \\
\hline $\begin{array}{l}\text { 7. ¿La organización controla la información que se comunica a sus } \\
\text { asociados de negocios? }\end{array}$ & $30 \%$ & $70 \%$ \\
\hline $\begin{array}{l}\text { 8. ¿Considera Ud. que la información que dispone la empresa está } \\
\text { organizada y representada de forma correcta para los diversos públicos, } \\
\text { clientes o usuarios? }\end{array}$ & $90 \%$ & $10 \%$ \\
\hline $\begin{array}{l}\text { 9. ¿Se dispone de una política informacional incluida en sus normativas } \\
\text { internas sobre el uso y manejo de la información? }\end{array}$ & $70 \%$ & $30 \%$ \\
\hline $\begin{array}{l}\text { 10. ¿La empresa cuenta con estrategias informacionales de desarrollo de } \\
\text { la actividad informacional? }\end{array}$ & $60 \%$ & $40 \%$ \\
\hline $\begin{array}{l}\text { 11. ¿Los flujos informacionales garantizan disponer de información de } \\
\text { calidad para su toma de decisiones en la empresa? }\end{array}$ & $80 \%$ & $20 \%$ \\
\hline $\begin{array}{l}\text { 12. ¿Tienen identificados indicadores para evaluar la actividad } \\
\text { informacional? }\end{array}$ & $50 \%$ & $50 \%$ \\
\hline 13. ¿La empresa verifica las redes de comunicación externa generada? & $80 \%$ & $20 \%$ \\
\hline 14. ¿Considera que son adecuados sus canales de comunicación externa? & $90 \%$ & $10 \%$ \\
\hline $\begin{array}{l}\text { 15. ¿Considera que se controlan los mensajes enviados a los asociados de } \\
\text { negocios? }\end{array}$ & $90 \%$ & $10 \%$ \\
\hline $\begin{array}{l}\text { 16. ¿Considera que para el control de la comunicación cuenta con personal } \\
\text { adecuado? }\end{array}$ & $100 \%$ & $0 \%$ \\
\hline $\begin{array}{l}\text { 17. ¿Cree Ud. que aplica la mejora continua en el control y evaluación de la } \\
\text { comunicación organizacional? }\end{array}$ & $50 \%$ & $50 \%$ \\
\hline $\begin{array}{l}\text { 18. ¿Considera Ud. Importante, integrar el uso de la información en los } \\
\text { elementos de la comunicación? }\end{array}$ & $50 \%$ & $50 \%$ \\
\hline $\begin{array}{l}\text { 19. ¿Cree Ud. que la política institucional debe manifestar coherencia entre } \\
\text { los procesos comunicacionales y la calidad de la información? }\end{array}$ & $100 \%$ & $0 \%$ \\
\hline $\begin{array}{l}\text { 20. ¿Considera que la empresa verifica el uso y comprensión de la } \\
\text { información que se comunica a los asociados de negocios que forman parte } \\
\text { de la cadena de suministros del comercio exterior? }\end{array}$ & $30 \%$ & $70 \%$ \\
\hline
\end{tabular}

Fuente: Los Autores (2021). 
En relación con la gestión de información, se nota en los datos recabados que apenas el $20 \%$ de las empresas que constituyen la cadena de suministros del comercio internacional cuentan con un sistema de información que controla las necesidades y fuentes de información relevantes, el $80 \%$ dicen no tenerlo. Así mismo el $20 \%$ señalan que consideran en sus procesos las características de uso adecuado de la información, el $80 \%$ no lo hacen. En referencia al control de la información que se entrega a sus asociados de negocios el $80 \%$ creen controlarla a diferencia del $20 \%$ que dice no hacerlo. Se observa que el $50 \%$ de las empresas dicen que su personal está capacitado para manejar la información generada para la cadena de suministros de la exportación y el otro $50 \%$ no.

Al analizar el programa de capacitación planificado, indican que consideran el conocimiento de competencias informacionales en el desarrollo del personal de la empresa un $20 \%$, mientras que el $80 \%$ restante no lo considera. Un $20 \%$ de las empresas, revisan los resultados de la Gestión informacional a diferencia del $80 \%$ que no lo hace. Si se considera que la organización controla la información que se comunica a los asociados de negocios, señalan el $30 \%$ que lo hace y el $70 \%$ no. Afirman el $90 \%$ que, la información que dispone la empresa está organizada y representada de forma correcta para los diversos públicos, clientes o usuarios y apenas el $10 \%$ reconoce dice que no.

Al preguntar si se dispone de una política informacional incluida en la normativa para el uso y manejo de la información reconocen el $70 \%$ que, si lo tienen, mientras que el $30 \%$ dice no tenerlo. Consecuentemente a lo anterior el $60 \%$ dice contar con estrategias informacionales de desarrollo de la actividad informacional y el $40 \%$ no lo tiene. Al referirse a que si los flujos informacionales garantizan disponer de información de calidad dicen el 80\% que los consideran para la toma de decisiones y el $20 \%$ no lo hace. Así también el $50 \%$ tienen identificados indicadores para evaluar la actividad informacional, 
frente al otro $50 \%$ que no.

Además de las preguntas dicotómicas analizadas sobre gestión de información, se pudieron recabar criterios sobre el tipo de información que les resulta esencial para el desarrollo de su trabajo en cuanto a documentos y archivos que consideran esencial dependiendo del tipo de empresa consultada, por lo que señalan las siguientes ideas:

a). Para exportar se necesita orden de compra, factura, lista de empaque, certificados de origen, pólizas, declaraciones aduaneras, etc.

b). Análisis de datos, indicadores, objetivos, cuadro de mando integral.

c). Páginas web, regulaciones aduaneras, regulaciones de comercio exterior.

d). Los documentos del contenedor y los permisos de movimiento del transporte y los documentos en regla de los choferes.

e). Reglamentos de la aduana y navieras.

f). La recopilación de información que arroja nuestra web, información de requisitos aduaneros mundiales.

g). Es esencial que las normas estén claras y documentadas y que hayan sido socializadas a todos los empleados para luego poder replicar a nuestros clientes.

h). Los documentos que son presentados por los usuarios.

Con relación a la gestión de la comunicación, se observa que el $80 \%$ de empresas verifican las redes de comunicación externa frente al $20 \%$ que dice no hacerlo. Con ello se dice además que el $90 \%$ posee canales adecuados de comunicación con sus asociados de negocios y solamente el $10 \%$ no dispone. En referencia con el control de mensajes entregados, el $90 \%$ afirma realizarlo y apenas un $10 \%$ dice no hacerlo. Se nota que el $100 \%$ de las empresas en cuestión, disponen de personal adecuado en este campo ya que ninguno; $0 \%$ dijo no tenerlo. 
En cuanto al control y evaluación de la comunicación con miras a la mejora continua el criterio se divide $50 \%$ y $50 \%$ entre los encuestados; por lo que, concomitante a ello la organización cree importante que se pueda integrar el uso de la información con los elementos de la comunicación en ese mismo porcentaje $50 \%$ y $50 \%$. El $100 \%$ de los miembros de este estudio creen que la política institucional debe tener coherencia entre la calidad de información y los procesos comunicacionales, por lo que no hay contrariedad con el $0 \%$. Apenas el 30\% reconoce que su empresa verifica el uso y comprensión de la información comunicada a sus asociados de negocios, mientras que el $70 \%$ reconoce no hacerlo. En referencia a la comunicación organizacional, lo más relevante que debe ser utilizado por este tipo de empresas señalan lo siguiente:

a). Un buen canal de comunicación es básico, información confiable, directa y en línea.

b). Que establezcan los flujos de comunicación adecuados en las organizaciones.

c). Estatus de las cargas, siniestros, nuevas regulaciones, nuevos costos, cambios en itinerarios.

d). Los documentos que tienen la información de la carga a ser transportada

e). Información de aduanas y facturas y conocimientos de embarque.

f). Procedimientos, prácticas y políticas debidamente claras y definidas.

g). La información que es relevante para que se cumplan los procesos de comunicación organizacional radica en el correcto funcionamiento de la tecnología al interior de la organización y que la dirección tenga claro lo que desea trasmitir.

h). Que la información clave sea difundida oportunamente.

i). Que estén establecidos las actividades de cada departamento para que la información fluya oportunamente. 
Finalmente se debe considerar entonces que las empresas motivo de análisis, tienen integrados varios elementos informacionales y comunicacionales en la trazabilidad de la carga, pero no todos son expuestos en la redacción de los artículos normativos de BASC, ni se detallan en la gestión que debe controlar la alta dirección de las organizaciones de las empresas de comercio exterior.

\section{Conclusiones}

Con los datos investigados, se han podido sistematizar los conceptos teóricos y características particulares tanto de la gestión de información como de la gestión de la comunicación enfocados al comercio exterior. Así como analizar la aplicación de dichos conceptos en las empresas de la cadena logística que generan trazabilidad como se mencionaron; empresas productoras importadores y exportadores, transporte aéreo, terrestre y marítimo, agentes de carga, agentes afianzados, navieras y puertos.

De igual modo, se debieron analizar los conceptos de seguridad de la carga en el comercio exterior y sus vulnerabilidades con el propósito de definir bondades en la seguridad de información y la comunicación en los procesos del comercio exterior considerando las actividades ilícitas presentes en ellos como son: tráfico de drogas, tráfico de personas, corrupción, soborno, lavado de activos, etc.

Se ha visto teóricamente los elementos de información y los componentes de la comunicación en varias empresas de comercio exterior, que se tratan de manera aislada, consiguiéndose resultados parciales y es imprescindible que sean gestionados en conjunto y de forma complementaria, considerando a la gestión de información como un eslabón indispensable para la efectiva comunicación en el comercio exterior.

En los resultados conseguidos, se pudieron analizar a fondo los ejes transversales de la información y su incidencia en la comunicación efectiva 
dentro de las normas BASC, encontrando sus acápites como incompletos y en base a lo cual se deja como aporte innovador, una estructura que mejora la gestión de las empresas de comercio exterior, para que a futuro permitan al capítulo de estas normas internacionales, reforzar sus controles y vulnerabilidades, proponiendo a la alta dirección de dichas empresas que en la toma de decisiones se incluya como datos de entrada y elementos de salida; los siguientes:

- El análisis y control de los Indicadores de los procesos en tiempos establecidos.

- Gestionar las no conformidades y acciones correctivas productos de la auditoría interna y externa.

- Verificar el cumplimiento de los requisitos legales y reglamentarios.

- Incluir en la planificación estratégica institucional, objetivos informacionales y comunicacionales.

- Disponer de una política del manejo y uso de la gestión de información y comunicación dentro de las normativas internas.

- Contar con una estrategia informacional y comunicacional de desarrollo progresivo.

- Contar con un profesional con competencias adecuadas en la gestión de información y en la gestión de la comunicación que conste en el orgánico estructural.

- Contar con un programa de alfabetización informacional y comunicacional.

- Contar con un sistema de información y comunicación diseñado para controlar el flujo de información relevante; así como las redes de comunicación tanto interna como externa.

- Caracterizar los procedimientos informacionales y comunicacionales en el sistema de gestión que permitan: identificar, analizar, procesar, 
Instituto Internacional de Investigación y Desarrollo Tecnológico Educativo INDTEC, C.A.

DOI: https://doi.org/10.29394/Scientific.issn.2542-2987.2021.6.21.3.58-80

OAI-PMH: http://www.indteca.com/ojs/index.php/Revista Scientific/oai

Artículo Original / Original Article

almacenar, recuperar y difundir la información; así como, definir los medios, controlar los mensajes, su contenido y los productos comunicacionales.

- Analizar, identificar y controlar las fuentes informacionales para la búsqueda y adquisición de información y monitoreados los productos de la comunicación.

- Tener identificación, control y monitoreo de los servicios de información, así como de los impactos de la comunicación (Auditoría).

- Tener control sobre la seguridad de la información y sus procedimientos de valor agregado a partir de los procesos organizacionales que se desarrollan los cuales deben ser comunicados a tiempo.

- Asegurar la calidad de la información y su incidencia en la comunicación considerando la mejora del desarrollo organizacional de las empresas de comercio exterior.

Como se ha podido notar producto de la recolección de la información, las empresas motivo de análisis, tienen integrados varios elementos informacionales y comunicacionales en la trazabilidad de la carga, pero no todos son expuestos en la redacción de los artículos normativos de la norma internacional BASC, ni se detallan en la gestión que debe controlar la alta dirección de las organizaciones de dichas compañías de comercio exterior, viéndose incompletos, los controles para la toma de decisiones y pudiendo ser razón de la materialización de los riesgos en función de actividades ilícitas, por lo que es importante integrar la propuesta presentada en este trabajo investigativo, en una futura revisión de las normas estudiadas e inclusive considerarse en otras normas semejantes que existen en el medio de estandarización. 


\section{Referencias}

BASC (2017). Norma Internacional BASC: Sistema de Gestión en Control y Seguridad. Miami, Florida, Estados Unidos: World BASC Organization, Business Alliance for Secure Commerce, BASC.

Fernández, L., \& Useche, M. (2015). Identidad y alteridad en la comunicación organizacional. Quórum Académico, 12(1), 60-77, eISSN: 1690-7582. Recuperado de:

https://www.redalyc.org/articulo.oa?id=199040067005

Medina, J. (2019). Gerencia Tecnológica de la Información y Comunicación en las Organizaciones Sociales. Revista Scientific, 4(12), 275-289, e-ISSN: 2542-2987. Recuperado de:

https://doi.org/10.29394/Scientific.issn.2542-2987.2019.4.12.14.275$\underline{289}$

Ponce, J. (ed.). (2005). Comercio exterior: alternativas para Ecuador. 1ra. Edición, ISBN: 9978-22-510-2. Quito, Ecuador: ABYA-YALA.

Gomes, C., \& Pomim, M. (2010). Información en el ámbito empresarial: una reflexión acerca de los modelos de gestión de la información. Ibersid: Revista de Sistemas de Información y Documentación, 4, 117121, e-ISSN: 1888-0967. Recuperado de:

https://www.ibersid.eu/ojs/index.php/ibersid/article/view/3828

Quintero, N., Giraldo, M., Bernal, I., Viana, C., \& Taborda, J. (2009). Identificación de las ciencias de la información documental. Revista Interamericana de Bibliotecología, 32(2),195-229, e-ISSN: 0120-0976. Recuperado de:

https://www.redalyc.org/articulo.oa?id=179016347007

Rodríguez, Y. (2015). Gestión de Información y del Conocimiento para la toma de decisiones organizacionales. Bibliotecas. Anales de investigación, 11(11), 150-163, e-ISSN: 1683-8947. Recuperado de: https://dialnet.unirioja.es/servlet/articulo?codigo $=5704545$ 
Huesca, C. (2012). Comercio Internacional. ISBN: 978-607-733-004-2. México: Red Tercer Milenio, S.C.

Rueda, I., Tamayo, G., Acosta, B., Cueva, F., \& Idrobo, P. (2020). Aprendizaje organizacional y su vinculación con la comunicación. SaberEs, 12(1), 73-85, e-ISSN: 1852-4222. Recuperado de:

http://dx.doi.org/10.35305/s.v12i1.174

Sánchez-Castellano, D., Rodríguez-Cruz, Y., \& Río-López, Y. (2018). Gestión de información y percepción organizacional en el Centro Nacional de Información de Ciencias Médicas de Cuba. Revista Cubana de Información en Ciencias de la Salud, 29(4), 1-15, e-ISSN: 2307-2113. Recuperado de:

http://www.rcics.sld.cu/index.php/acimed/article/view/1252

Salvatore, D. (2005). Economía Internacional. 8va. Edición, ISBN: 9789681862336. México: Editorial Limusa. 


\section{César Raúl Méndez Carpio \\ e-mail: cmendezc@ucacue.edu.ec}

Nacido en Cuenca, Ecuador, el 22 de febrero del año 1971.

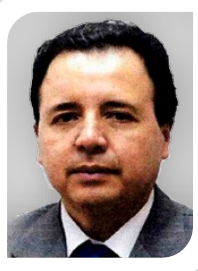

Dr. en Ciencias de la Educación, especialidad Ordenadores en la Universidad Católica de Cuenca (UCACUE); Lcdo. en Ciencias de la Educación, especialidad Ordenadores en la Universidad Católica de Cuenca (UCACUE); Magister en Docencia y Currículo para la Educación Superior en la Universidad Técnica de Ambato (UNITA); Magister en Administración de Empresas en la Universidad del Azuay (UDA); actualmente Jefe de Investigación en el Colegio Militar "Abdón Calderón" y docente titular en la Universidad Católica de Cuenca (UCACUE); acreditado como Investigador en la Secretaría de Educación Superior, Ciencia, Tecnología e Innovación (SENESCYT) con el No. REG-INV-21-04948. 


\section{Yunier Rodríguez Cruz \\ e-mail: yunier@fcom.uh}

Nacido en La Habana, Cuba, el 10 de noviembre del año

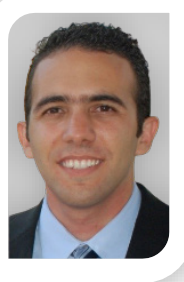
1982. Dr.(c). en Documentación e Información Científica por la Universidad de Granada (UGR), España; y por la Universidad de La Habana (UH), Cuba; MSc. en Bibliotecología y Ciencia de la Información por la Universidad de La Habana; Licdo. en Bibliotecología y Ciencias de la Información por la Facultad de Comunicación de la Universidad de La Habana; actualmente me desempeño como Profesor Titular del Departamento de Ciencias de la Información de la Facultad de Comunicación de la Universidad de La Habana; Docente e investigador en temas de Gestión de Información, Inteligencia Organizacional e Información para la toma de decisiones. 
OAI-PMH: http://www.indteca.com/ojs/index.php/Revista Scientific/oai

Artículo Original / Original Article

\section{Hilda María Saladrigas Medina \\ e-mail: saladrigas@fcom.uh}

Nacida en La Habana, Cuba, el 1 de marzo del año 1965.

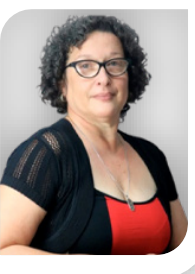

Dra. en Ciencias de la Comunicación Social por la Facultad de Comunicación de la Universidad de La Habana (UH), Cuba; Máster en Ciencias de la Comunicación por la Universidad de La Habana; Licda. en Filosofía Marxista Leninista de la Universidad de Oriente (UO), Santiago de Cuba; actualmente me desempeño como Profesora Titular y Decana de la Facultad de Comunicación de la Universidad de La Habana, Cuba, en Ciencias de la Comunicación social; Investigadora de Medios de Comunicación y Opinión pública; Miembro de la Academia de Ciencias de Cuba, de la Asociación Cubana de Comunicadores Sociales, del Ministerio de Educación Superior, de la Universidad de Oriente, de la Universidad de La Habana y de la Unión de Periodistas de Cuba.

El contenido de este manuscrito se difunde bajo una Licencia de Creative Commons ReconocimientoNoComercial-Compartirlgual 4.0 Internacional 